\title{
Editorial
}

Digestion

Published online: September 4, 2018

\section{Pancreaticobiliary Maljunction: Markedly High Risk for Biliary Cancer}

\author{
Terumi Kamisawa ${ }^{a}$ Goro Honda ${ }^{b}$ \\ ${ }^{a}$ Department of Internal Medicine, Tokyo Metropolitan Komagome Hospital, Tokyo, Japan; ${ }^{b}$ Department of Surgery, \\ Tokyo Metropolitan Komagome Hospital, Tokyo, Japan
}

The main pancreatic duct and the common bile duct open into the duodenum either separately or at one opening with or without a common channel. Common channel formation is reported to occur in $55 \%$ of cases [1]. The average length of the common channel has been reported to be $4.4 \mathrm{~mm}$ with a range of $1-12 \mathrm{~mm}$ [2]. The sphincter of Oddi, which is normally located at the distal end of the pancreatic and bile ducts, regulates the outflow of bile and the pancreatic juice.

Pancreaticobiliary maljunction (PBM) is a congenital malformation in which the junction of the pancreatic and bile ducts is located outside the duodenal wall. PBM can be divided into 2 types: with biliary dilatation (congenital biliary dilatation; Fig. 1) and without biliary dilatation (Fig. 2). PBM is diagnosed when an abnormally long common channel is evident on direct cholangiography such as endoscopic retrograde cholangiopancreatography or magnetic resonance cholangiopancreatography [3].

Since the pancreaticobiliary junction is not affected by the activity of the sphincter, regurgitation can occur in both directions: pancreatic juice can regurgitate into the common bile duct (pancreatobiliary reflux), and bile can

\section{KARGER}

๑) 2018 S. Karger AG, Basel

E-Mail karger@karger.com

www.karger.com/dig regurgitate into the pancreatic duct (biliopancreatic reflux). In PBM, because hydropressure is usually higher in the pancreatic duct than in the bile duct, pancreatic juice frequently refluxes into the biliary duct $[3,4]$. The ongoing presence of refluxed pancreatic juice in the biliary duct results in injury to biliary tract epithelium, which promotes cancer development; thus, patients with PBM have higher rates of carcinogenesis within the biliary tract [4]. A nationwide survey in Japan [5] showed that the prevalence of biliary tract cancers in adult PBM patients with and without biliary dilatation was 21.6 and $42.4 \%$ respectively. In PBM patients with biliary tract cancers, bile duct and gallbladder cancers were present in 32.1 and $62.3 \%$ of patients with biliary dilatation and in 7.3 and $88.1 \%$ of those without biliary dilatation respectively. Furthermore, biliary tract cancers developed at an early age in PBM patients (mean age, 50-60 years), about 1520 years earlier than in those without PBM [4].

In our series of $145 \mathrm{PBM}$ patients, bile duct cancer and gallbladder cancer occurred in $8(11 \%)$ and $15(21 \%)$ of 73 patients with congenital biliary dilatation, and in 2 (3\%) and $49(68 \%)$ of 72 patients with PBM without biliary dilatation respectively. The markedly high rate of as- 


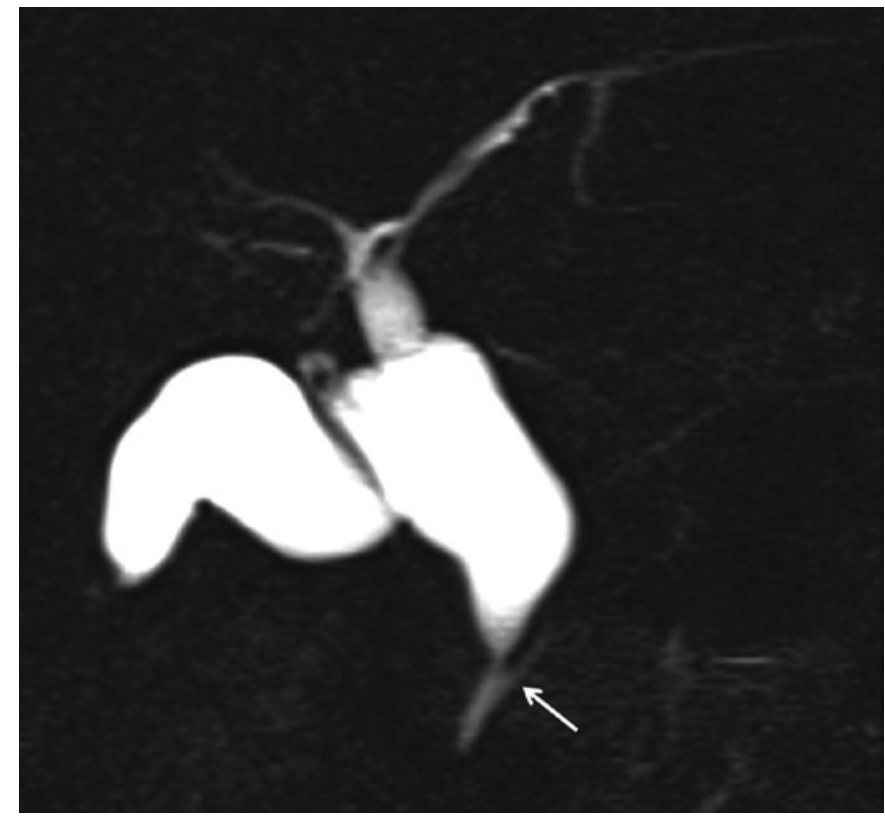

Fig. 1. MRCP of congenital biliary dilatation (arrow: PBM). MRCP, magnetic resonance cholangiopancreatography; PBM, pancreaticobiliary maljunction.

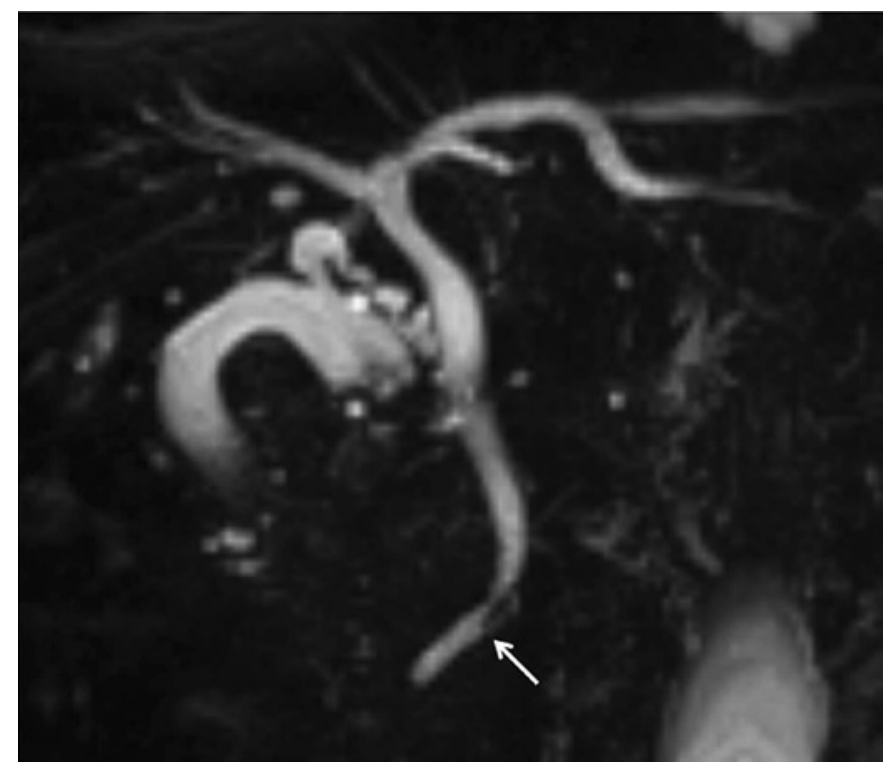

Fig. 2. $M R C P$ of $P B M$ without biliary dilatation (arrow: $P B M$ ). $\mathrm{MRCP}$, magnetic resonance cholangiopancreatography; PBM, pancreaticobiliary maljunction.

sociation of biliary cancer with PBM might be due to selection bias, as our hospital is the cancer center. Simultaneous or metachronous double cancers are sometimes seen in biliary cancers associated with PBM. The prevalence of gallstone detection in our 49 PBM patients with- out biliary dilatation who developed gallbladder cancer was only $10 \%$, which is significantly lower than that among the gallbladder cancer population without PBM (about 60\%).

The carcinogenetic mechanism in patients with PBM appears related to the stagnation of the pancreatic juice that is refluxed into the biliary tract. Refluxed proteolytic pancreatic enzymes and phospholipase A2 become activated in the biliary tract, resulting in the production of strong cytotoxic substances such as lysolecithin. The resultant chronic inflammation causes repeated cycles of damage to and healing of the biliary mucosal epithelia. As a result of these changes in the mucosal epithelia, in association with DNA mutations, cancer development and progression are ultimately promoted. The predominant mechanism responsible for the development of biliary tract cancer in patients with PBM appears to be the hyperplasia-dysplasia-carcinoma sequence, which is thought to differ from the predominant mechanisms seen in patients without PBM, which are the adenoma-carcinoma sequence and the de-novo carcinogenesis [4, 6-8].

Once the diagnosis of PBM is made, patients are counseled to have immediate prophylactic surgery prior to the development of malignant changes in the biliary tract. The standard surgical procedure for congenital biliary dilatation is extrahepatic bile duct resection with bilioenteric anastomosis, in which both the common bile duct and gallbladder, where malignancy can develop, are excised; this procedure also prevents both bile stasis and reflux of pancreatic juice into the bile duct. Since progression to cancer might occur in the remnant bile duct mucosa, it is necessary to perform extended or total biliary tract excision. Since hepatic duct transection can result in intrahepatic calculus formation, recurrent cholangitis, and cholangiocarcinoma, one must perform extended bile duct resection, including the resection of strictures of the hepatic or intrahepatic bile ducts. Dissection of the pancreatic portion of the common bile duct at the level immediately above the pancreaticobiliary junction is needed because postoperative carcinogenesis, pancreatitis, and pancreatic stones occur with a remnant bile duct $[4,7]$.

On the other hand, there is considerable debate about the treatment of PBM without biliary dilatation in patients without cancer. In many institutes, prophylactic cholecystectomy is performed because most biliary cancers in PBM patients without biliary dilatation are gallbladder cancers. However, some surgeons suggest that the extrahepatic bile duct should be removed together with the gallbladder in PBM patients without biliary dil- 
atation because such patients have a higher risk of bile duct cancer than the general population, and they also reported to have K-ras and/or p53 gene mutations in the bile duct [9]. This is a particularly important tissue in children with PBM, since pancreatic secretions continue to come into contact with the bile during their long lifespan $[4,7]$.

Making the diagnosis of PBM before the onset of biliary carcinogenesis and then performing a prophylactic surgery are vital. Unlike patients with congenital biliary dilatation, patients with PBM without biliary dilatation rarely have symptoms, so that the majority of such patients remain undiagnosed until they present with advanced-stage gallbladder cancer. The characteristic pathological change seen in PBM patients is epithelial hyperplasia of the gallbladder due to longstanding continuous stasis of the bile intermixed with refluxed pancreatic juice [4]. In order to identify PBM without biliary dilatation at an early stage, it is important to perform magnetic resonance cholangiopancreatography for patients with gallbladder wall thickening on screening ultrasonography under suspicion of PBM $[8,10]$.

In conclusion, $\mathrm{PBM}$ is a congenital malformation with a markedly high risk for biliary cancer. Once PBM is diagnosed, appropriate prophylactic surgery should be performed immediately. Since most PBM patients without biliary dilatation present with advanced gallbladder cancer, we should develop a new strategy for the early diagnosis of PBM.

\section{Acknowledgments}

This work was supported by a grant from the Ministry of Health, Labor, and Welfare of Japan (H28-nanchitou(nan)-ippan-021, Research on Rare and Intractable Diseases, Health and Labor Sciences Research Grants.

\section{References}

1 Sterling JA: The common channel for bile and pancreatic ducts. Surg Gynecol Obstet 1954; 98:420-424.

2 Dowdy GS, Waldron GW, Brown WG: Surgical anatomy of the pancreatobiliary ductal system. Observations. Arch Surg 1962;84: 229-246.

3 Kamisawa T, Ando H, Hamada Y, Fujii H, Koshinaga T, Urushihara N, Itoi T, Shimada H; Japanese Study Group on Pancreaticobiliary Maljunction: Diagnostic criteria for pancreaticobiliary maljunction 2013. J Hepatobiliary Pancreat Sci 2014;21:159-161.

4 Kamisawa T, Ando H, Suyama M, Shimada M, Morine Y, Shimada H; Working Committee of Clinical Practice Guidelines for Pancreaticobiliary Maljunction: Japanese clinical practice guidelines for pancreaticobiliary maljunction. J Gastroenterol 2012;47:731759.

5 Morine Y, Shimada M, Takamatsu H, Araida T, Endo I, Kubota M, Toki A, Noda T, Matsumura T, Miyakawa S, Ishibashi H, Kamisawa $\mathrm{T}$, Shimada H: Clinical features of pancreaticobiliary maljunction: update analysis of $2 \mathrm{nd}$ Japan-nationwide survey. J Hepatobiliary Pancreat Sci 2013;20:472-480.

6 Shimada K, Yanagisawa J, Nakayama F: Increased lysophosphatidylcholine and pancreatic enzyme content in bile of patients with anomalous pancreaticobiliary ductal junction. Hepatology 1991;13:438-444.

7 Kamisawa T, Kaneko K, Itoi T, Ando H: Pancreaticobiliary maljunction and congenital biliary dilatation. Lancet Gastroenterol Hepatol 2017;2:610-618.

8 Kamisawa T, Kuruma S, Chiba K, Tabata T, Koizumi S, Kikuyama M: Biliary carcinogenesis in pancreaticobiliary maljunction. J Gastroenterol 2017;52:158-163.

9 Matsubara T, Sakurai Y, Zhi LZ, Miura H, Ochiai M, Funabiki T: K-ras and p53 gene mutations in noncancerous biliary lesions of patients with pancreaticobiliary maljunction. J Hepatobiliary Pancreat Surg 2002;9:312-321.

10 Takuma K, Kamisawa T, Tabata T, Hara S, Kuruma S, Inaba Y, Kurata M, Honda G, Tsuruta K, Horiguchi S, Igarashi Y: Importance of early diagnosis of pancreaticobiliary maljunction without biliary dilatation. World J Gastroenterol 2012;18:3409-3414. 